\title{
Course 2 - creating a specialty registrars procedure course
}

\author{
Authors: Oliver O'Sullivan ${ }^{\mathrm{A}}$ and Amanda Laird ${ }^{\mathrm{B} *}$
}

\section{Introduction}

I am a Royal College of Physicians associate college tutor at Queen Alexandra Hospital, Portsmouth. As part of this role, one of my responsibilities is to deliver the Portsmouth Procedure Course, designed to offer simulation training in core curriculum skills to core medical and acute care common stem trainees. The course, tutored by subject matter experts (consultants or senior member of the multidisciplinary team), combined classroom theory with skills lab practical sessions.

I noticed that specialty registrars (SpRs) often applied to the course to perform procedural skills that weren't part of their core job role, and I felt there was a gap in their educational provision. I designed an SpR Procedures Course, focusing on the revision of the procedural skills, but also, recognising the senior level of the trainees, how to manage complications.

\section{Materials and methods}

The aspiration was for the new course to be peer led, with SpRs teaching fellow SpRs, giving all members of the course a chance to share their own expertise and ask questions of real life situations, creating an equal learning and sharing environment (with added benefits of developing clinical teaching skills and interdepartmental relationships). We ran two pilot courses to hone content and timings.

\section{Results and discussion}

Key differences from the SHO course was minimal classroom time (a brief revision session) with increased time in the skills lab to perform the procedure (as all SpRs had knowledge of procedural skills) and a Q\&A at the end of each session for any on call emergencies / real-life situations.

Costs have been minimal (approximately $£ 200$ ) due to the faculty being organic, with the bulk of costs being the consumables. We feel this is excellent value for improving the education and support for our medical registrars, especially given the formal introduction of simulation training in the updated curriculum.

\section{Conclusion}

Feedback was universally positive, with the trust commissioning multiple courses a year to meet the demand. The SpRs felt appreciated and were delighted that a course had been designed with them in mind. Over the next year, we aim to integrate this course with a cardiac arrest team-skills simulation course, extend pleural procedure element, and share the course with the Wessex Simulation Network as an example of good practice.

\section{Conflicts of interest}

None declared.

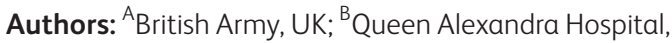
Portsmouth, UK; *RCP chief registrar 